\title{
Examining User Experience of Cruise Online Search Funnel
}

\author{
Asta Adukaite ${ }^{1}$, Alessandro Inversini ${ }^{2}$, and Lorenzo Cantoni ${ }^{1}$ \\ ${ }^{1}$ Università della Svizzera italiana, Lugano, Switzerland \\ ${ }^{2}$ Bournemouth University, United Kingdom \\ \{asta.adukaite, lorenzo.cantoni\}@usi.ch, \\ ainversini@bournemouth.ac.uk
}

\begin{abstract}
While the cruising industry is enjoying significant and stable growth rates, its online communication has not been extensively researched. This may be due to the fact that cruises are still being sold mainly offline. Nonetheless, both cruisers and the industry itself are changing, and the role played by online communication is getting more and more importance. By adopting a user testing approach, this paper presents results of an analysis done on the online search funnel of the four main cruising companies. Such analysis is useful not only to compare the usability of the selected websites, but also to better define critical aspects of the search funnel within cruise websites from the viewpoint of user experience.
\end{abstract}

Keywords: Usability, e-Tourism, cruise websites.

\section{Introduction}

The intense development and evolution of technologies in the tourism field led to an extreme specialization of the online communication within the sectors by which tourism is composed [3] not only in terms of design and development of new technology and business models, but also in terms of quality [12] of the online communication itself. The advent of technologies in the tourism arena brought a series of advantages not only within the marketing [4] and the operations management [21] of tourism destinations, attractions and industries connected to tourism, the advantages can be recognized also within the purchase processes that radically changed in the last decades [25]. Purchase process and all the related aspects have been mostly discussed in a specific field that is the one related to accommodation and hotels [19]. Little research to date concentrates on different fields such as the one presented and discussed in this paper - the cruise sector. The cruise sector experienced a huge transformation in the last decade and has changed from a luxury activity to an affordable type of vacation [15]. Even if most of the cruises are sold by travel agents [7] it is emerging in the literature [20] but also in the industry, the possibility of employing new technologies not only for marketing activities but also for selling purposes. 


\section{Literature}

\subsection{ICT in Cruising Industry Domain}

The cruising industry is experiencing stable and solid growth regardless its short history. The demand for cruising vacations is growing by a remarkable pace, new ships are being built every year with innovative style and design offering abundance of new products and services attempting to enhance cruising experience. Once a leisure luxury activity for rich people, the cruising vacation has transformed to an affordable type of vacation to many people nowadays [15]. In 1990 the number of passengers was around 3.7 million, in 2012 there were already 20.1 million, with an annual growth of approximately $7.38 \%$ [6]. The estimations by the industry are reporting a continues growth of the cruise market, reaching by 201522.4 million, and by 2020 even 27 million of passengers [6]. While tourism organisations mostly use the web to market and sell their products, within the cruise industry the online distribution channel is still playing a minor role. Traditionally cruise operators focus on cruise package sales through the intermediaries - namely travel agents; about $74 \%$ cruisers book at least a certain part of their cruises with travel agents [7]. However, taking into consideration the revolution of ICT and emergence of eTourism, there is an interesting question uprising concerning the effectiveness of the online distribution channel within the cruise sector. According to Papathanassis and Breitner [20] the cruise product is perceived as complex, expensive and purchased by older people and as a consequence the internet as a distribution channel is considered not effective. In this case human agents are in a better position to manage the amount of necessary information and to deal with emotional demands of the cruisers. The CEO of Expedia, D. Khosrowshahi [23] has acknowledged that "cruise product lends itself to a more hands-on sale...the more complex the product the more difficult it is to sell that product online." Top executives of Expedia and Priceline have stated that "despite the ease of online travel bookings and the wealth of helpful travel reviews, cruise buyers still want a flesh-and-blood travel agent to help them plan those trips" [23]. Nevertheless, over the past years, cruising experience and the passengers have changed considerably as cruise product is becoming cheaper, standardized and accessible to big numbers of travellers. According to CLIA (Cruise Lines International Association), 2010 Cruise Market Profile Study [7], the average cruiser's profile is 45 years old with $\$ 79,000$ - $\$ 93,000$ annual earnings, $65 \%$ have an higher education diploma, and only $15 \%$ are retired while others are professionally active. Hence, the characteristics of a current average cruiser strongly contradict the stereotypical perception of cruisers being old and internet-illiterate.

It is possible then to argue that, the proliferation and emergence of new travel distribution channels triggered by the progress of the internet has offered cruisers the alternative way to book the cruise vacations. Moreover, looking at the cruiser's profile there is a good justification for internet as a cruise distribution channel, however, it seems that its adoption is still limited [20]. 


\subsection{User Experience and Usability}

According to Garrett [9], user experience is not about how a product works on the inside (although that sometimes has a lot of influence), but it is about how it works on the outside, where a person comes into contact with it and has to work with it. The same author described the website as a "self-service product", where no instruction manual or seminar is provided: users face the website alone, only with their experience guiding them [9]. The quality assessment in the tourism field has been widely investigated both at destination level [11] and at the hospitality level [26]. Different methodologies and strategies have been used to assess quality and usability of online applications in tourism: human computer interaction related methodologies (i.e. expert review and user testing [11]), methods derived from technology acceptance models, as well as users' assessment of functionality and usability attributes have been also adopted in the field [22]. In some cases, given the complexity [21] and the importance of the tourism online communication [4], the specific field of research also led to the creation and testing of new analysis models, able for example to connect usability and user experience with usages [12]. This research considers the definition given by ISO (International Organisation for Standardization) which defines usability (ISO 9241) as "the effectiveness, efficiency and satisfaction with which specified users achieve specified goals in particular environments", thus putting the users in a central position within the assessment of the quality of a website.

The paper analyses and discusses the usability and quality of the online search funnel experience of four cruise websites, leveraging on a methodology used extensively in usability testing both for heuristic drive evaluation and for user testing, namely MiLE+ [24]. Search funnel is a crucial issue for the online communication of cruising companies, even if the majority of actual bookings are done offline [7], the study of this funnel is relevant to assess online mechanisms that may influence the choice of the cruise package to be booked via travel agent. Furthermore, investigating and comparing these particular sets of functionalities in cruising websites, understanding their shortcomings and recording users' opinions will shed lights on the possible improvements, eventually leading to actual online booking. It is important to note that this paper considers only the search funnel of these websites, it does not analyse the payment and check out process.

\section{Research Design}

The main objective of this research is to understand the usability and the user experience of the online search funnels of cruise websites (focusing on Business to Consumer side), and to find out what kind of user experiences shortcomings and usability issues are present that might affect negatively user experience, and concur in dissuading conversion.

A scenario-based evaluation (i.e. definition of user profiles, goals and tasks [5]) was performed in order to define how a website will be approached and used by real users and how it will serve them to fulfil specific needs in explicit situations by completing typical and significant activities within the website, also taking into consideration the 
satisfaction levels of the users when interacting with the website [24]. Different scenarios were defined thanks to semi-structured interviews with experts from the field (representatives from the cruising industry), and reverse-design activities performed on the websites, in order to understand their business objectives. To ensure that users profiles, goals and tasks were realistic and relevant, researchers used also insights from secondary data, namely: (i) Alexa.com audience profiles; (ii) www.cruisecritic.com members' reviews; (iii) 2010 Traveler's Road To Decision - Cruise, [10] and (iv) Reports from Cruise Lines International Association [7].

The sample of cruise operators was selected analysing the statistics of Cruise Market Watch and focusing on the share of world wide passengers carried by cruise lines as well as passenger capacity. According to current data Carnival and RCCL (Royal Caribbean Cruise Line) are undoubtedly the biggest players in the cruise market arena in terms of both capacity (Carnival $21.1 \%$, RCCL $16.9 \%$ ) and carried passengers (Carnival 14.6\%, RCCL 14.6\%), therefore, these two cruise lines were chosen for the empirical testing. The other two cruise lines selected for analysis were NCL (Norwegian Cruise Line) and MSC Cruises because they also occupy a big share of market (NCL 7.1\%, MSC 5.8\%) and capacity (NCL 6.2\%, MSC 6.5\%). Important to note that Princess Cruises (market share 6.4\%, capacity 8.6\%) and Costa Cruises (market share $7.2 \%$, capacity $8.6 \%$ ) have a similar market share and passenger capacity, however, Princess and Costa belong to Carnival Corporation and the biggest player in the group Carnival Cruise Lines has already been chosen to be analysed. Meanwhile NCL and MSC Cruises do not belong to the two main groups of cruise companies, MSC Cruises is a privately own company and NCL is jointly owned by Genting Hong Kong and Apollo Management, therefore, the selected sample is representative of the main players in the worldwide cruising industry arena.

\subsection{User Scenarios Elicitation and Evaluation}

The tasks presented in the scenario below (table 1) are the most common tasks of all scenarios drawn and together can be considered as a "meta scenario", or in other words the common core of each tested scenario. The scenario based evaluation was performed asking the users to navigate the website and complete or attempt to complete all 11 tasks in order to achieve the overall goal. Each user had to complete the same set of tasks and achieve the goal on all four selected cruise websites, which have been presented in a random order.

The goal of evaluator was to assess the usability and the user experience of the selected users towards the online cruise search funnels of the chosen cruise companies. Particularly, three research goals were defined to assess the general user experience of the testers:

1. The ability of the users to perform a specific task.

2. The amount of time needed to perform the given tasks.

3. The level of satisfaction in performing the tasks. 
Table 1. Meta Scenario

\begin{tabular}{l|l} 
SC1 & Meta scenario \\
\hline GOAL & $\begin{array}{l}\text { To find a cruise offer for a minimum of two adults in Caribbean for } 7 \text { nights in March } 2012 \\
\text { as well as all the relevant information concerning the services on-board and proceeding for } \\
\text { booking the cruise online or contacting travel agent. }\end{array}$ \\
\hline \multirow{2}{*}{ TASKS } & $\begin{array}{l}\text { T1: Find the cruise search tool and insert the above specified criteria. } \\
\text { T2: Sort the results by price. } \\
\text { T3: Select the cheapest } 2 \text { offers and compare them. } \\
\text { T4: Select one of them and click for more details. } \\
\text { T5: Explore the itinerary obtaining the information about the ports of call. } \\
\text { T6: Find the list of excursions in the ports of call of the selected cruise itinerary. } \\
\text { T7: Find information about the difficulty levels of the excursions. } \\
\text { T8: Find information about the cabins (prices, factual information, amenities). } \\
\text { T9: Find the information about the ship of the offer (entertainment, venues etc.). } \\
\text { T10: Save the selected cruise itinerary. } \\
\text { T11: Proceed for booking this offer online or contact travel agency to buy this package. }\end{array}$
\end{tabular}

In order to achieve this, a severity scale [17] was adopted to rank results: point 1 was given when the task was fully completed, 0 when the user was not able to complete the task at all, and 0.5 when the task was only partially completed. A thinking aloud protocol [2] was used to gather the data from users during usability testing. The usability testing participants were encouraged to express their thoughts out loud (what they are looking at, thinking, doing, feeling) as they were performing a set of tasks. This technique enabled to observe the process of task completion and to realize the obstacles users were facing. The thinking aloud protocol was divided into two parts, the first one was simultaneous thinking aloud protocol collecting data during the tasks' performance, and the second part was a retrospective thinking aloud protocol congregated after the scenario performance [14]. At the end of the session (second part), participants were asked to give a retrospective look in order to measure their satisfaction and evaluate overall experience on all websites from 1 to 4 (1 being the lowest satisfaction level, and 4 the highest one). Researches recorded the sessions with users and took notes (e.g. task completion, reactions of users). User testing has been conducted from 5th to 9th of September 2011.

\section{$4 \quad$ Results}

The testing was performed by a sample of 11 users who volunteered. Demographically they were 3 males and 8 females, aged from 24 to 32 (average 27 years old). The selected users were considered as familiar with digital technologies and as a prospect cruisers have a high likelihood to search cruise package online.

In order to assess the ability of the users to perform each specific task (Research Goal 1), researchers measured the percentage of tasks' completion on each website. Results (Fig. 1.) demonstrate that users completed $91 \%$ of the tasks on the RCCL websites, $80 \%$ on NCL website, $70 \%$ on Carnival and $50 \%$ on MSC website. 


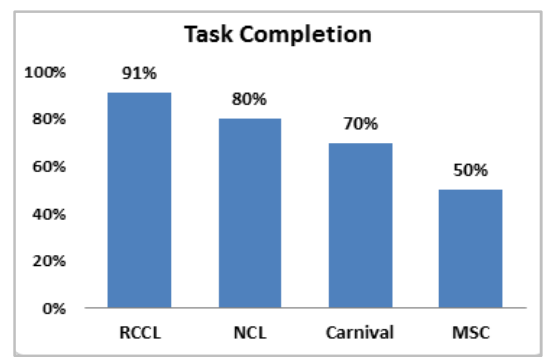

Fig. 1. Task completion

For what concerns the average time spent on a single website (Research Goal 2), researchers measured how much time for the given set of tasks users spent on each website (Fig. 2.). Overall results revealed that the longest to complete the tasks it took on MSC Cruises website - almost 12 minutes. However, it is important to highlight that longer time does not necessarily imply a negative user experience; for example, two users enjoyed navigating RCCL website (10.6 minutes), watching videos and getting additional information. This led to an increase of the time spent to complete the tasks, but most probably not to a reduced satisfaction level. Furthermore, the shortest time needed to conduct all the tasks was performed on NCL website (7.6 minutes).

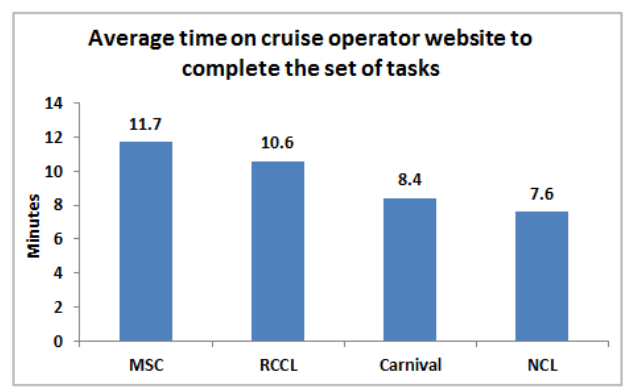

Fig. 2. Average time needed to complete the set of tasks

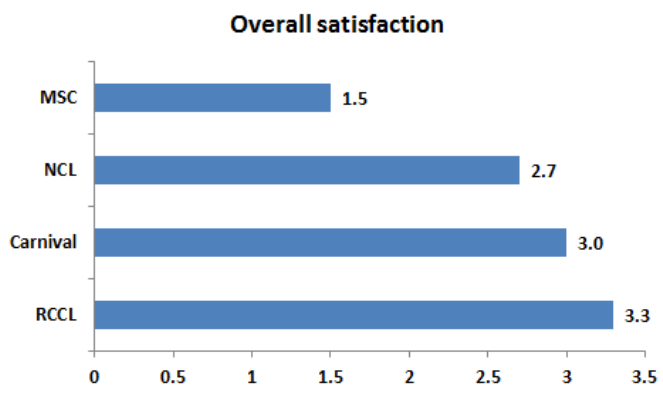

Fig. 3. Overall satisfaction, 4-level Likert scale 
Finally, in order to assess the overall satisfaction with the websites the evaluators asked participants to give a retrospective look to their experience, and to evaluate their overall satisfaction on a four-level Likert scale: the obtained results (Fig. 3.) demonstrate that users were mostly satisfied with RCCL (3.3), then Carnival (3.0), NCL (2.3), and the least with MSC (1.5).

The goal of the above described user based usability evaluation procedure was to define the most critical points in the cruise searching conversion funnel and observe where the users do not navigate easily and struggle the most. Overall, the following four critical issues were found within the user testing.

Search/Modification Functionality (7/11). 7 users commented about inflexibility of cruise online search in terms of required search criteria and filtering process. For instance, only NCL allowed users to insert the date range (from/to) for desired cruise, meanwhile the other 3 companies were constraining the users to select one single month at a time. Besides, 5 users expressed disappointment for the absence of an effective calendar management on the cruise lines websites. Furthermore, it was observed that users relied on a backward navigation, leading to the loss of information due to session expiry.

Results Pages (6/11). 6 users commented on the lack of easiness and flexibility to modify and manipulate the obtained (long) lists of results after inserting search criteria. Frequently the pages with obtained search results contained a big amount of information and in many instances the users struggled to understand the categorization of the results in order to proceed further. Carnival and RCCL offered quite extensive list of sorting criteria (price, duration, destination, ship, departure port, itinerary, date etc.), while NCL offered three sorting elements (best match, price and date), and MSC Cruises did not have sorting functionality and did not provide any indications according to which order the results were displayed.

Comparison Tools (6/11). 6 users commented on packages' "compare functionality" because from their visual representations they have struggled to understand what was actually being compared. 2 out of 4 cruise operators (RCCL and NCL) were offering packages comparing possibility, the feature of comparing was clearly displayed on cruise companies' websites and majority of the users were finding it easily and understanding how this functionality worked; however, once the comparison page was displayed they started doubting what exactly was being compared: prices, dates, ports, departure etc. due to overwhelming number of packages' details displayed at once.

Package Content Presentation in Condensed Manner (5/11). At the stage where users were dealing with a concrete selected package, 5 users commented on inability of the cruise operators to present the cruise package contents in a condensed and clear way. Furthermore, 9 users underlined the lack of clear pricing calculations of the packages, and inability to understand what exact charges were included in the price exposed, and what additional payments were supposed to be done on board. 


\section{Discussion and Conclusions}

Even though the websites' order in every single testing was randomized, researchers observed that there was a learning curve present (Fig 4.): this means that no matter which website was the first it took longer to complete the set of tasks (average: 12.5 minutes) than for the subsequent ones. The most significant difference was observed with the 1 st website, then the difference is not relevant among $2 \mathrm{nd}(8.8 \mathrm{~min})$, 3rd (8.8), and 4th (8.1 $\mathrm{min})$.

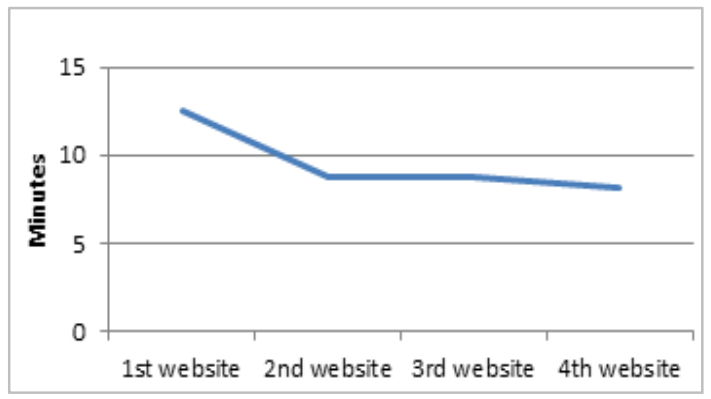

Fig. 4. Learning curve in user testing

\subsection{Critical Usability Issues and Suggestions for Improvement}

Several general conclusions or suggestions for improvement can be presented from the user testing outcome. Clear and well-designed 1st step for search is an essential feature, by displaying the start of the booking process (so called "quick search" box) on the homepage, cruise operators clearly communicate how users can begin their cruise vacation search. An effective calendar was acknowledged by users as a missing feature (this need might have been influenced by previous experiences with searching other touristic products: flights, hotels etc.), however, it does not seem the common feature currently on the cruise operators websites, only NCL offered this functionality, which was noticed by the users.

A well-organized result page is very important. When users are provided with a long list of cruising packages, it is crucial that they are be able to manipulate them easily. The possibility of sorting packages according to different criteria was acknowledged by users as an important functionality. Also the filtering functionality, which was clearly displayed by Carnival, was noticed by the users because it allowed to reduce the number of the provided cruise packages by excluding those that did not fit given criteria.

The "compare" functionality has to be clear in terms of what is actually being compared: prices, departure port, itinerary, ship, special offers etc. There should be always an alignment between the selected cruise offer and the provided pieces of information (e.g.: excursions, ports of call, booking etc.). Furthermore, it is also important to strike the right balance among all provided contents: because the cruise product is a very complex one, the amount of information and the way it is presented 
are critical aspects. While information overload might deter users, too generic or no information about specific elements might reduce credibility in the company.

"Memory" of users' inputs while a search is refined was acknowledged as fundamental, in case users are not fully satisfied, and want to perform a subsequent search, it is essential to provide an easy procedure, which saves the previously input search criteria (such as destination, date etc.). In fact, in all cases, when testers were clicking backward navigation button and were losing the already generated results and inserted search criteria it was provoking frustration from their side.

Majority ( 9 out of 11) of the testers were saying that display of transparent pricing is essential. As the cruise product is perceived as expensive, it is critical to provide as early as possible what is going to be included in the price and what are other additional charges. Two users proposed that price calculator or some kind of "drag and drop" functionality would help users to understand pricing of the cruise package.

\section{$6 \quad$ Limitations}

Usability testing was conducted with a sample consisting of young people, younger than the average cruiser, while this is a limitation in itself, it can also offer insights onto the approach by a public who is likely to become very interesting for the cruising companies in the future, and likely to consider not only online search, but also online cruise booking.

\section{References}

1. Boren, T., Ramey, J.: Thinking aloud: reconciling theory and practice. IEEE Transactions on Professional Communication 43(3), 261-278 (2000)

2. Brinck, T., Gergle, D., Wood, S.D.: Usability for the Web: Designing Web Sites that Work, 1st edn. Morgan Kaufmann, San Francisco (2001)

3. Buhalis, D., Law, R.: Progress in information technology and tourism management: 20 years on and 10 years after the Internet-The state of eTourism research. Tourism Management 29(4), 609-623 (2008)

4. Buhalis, D.: eTourism: Information technology for strategic tourism management. Prentice Hall, Harlow (2003)

5. Carroll, J.M.: Making use: scenario-based design of human-computer interaction. The MIT Press, Cambridge (2000)

6. Cruise Market Watch statistics, http://www. cruisemarketwatch.com/

7. CLIA (Cruise Lines International Association): Market Profile Study, Cruise Industry Economic Study, Consumer Cruise Lifestyle Trends Survey (2010)

8. Dix, A., Finlay, J.E., Abowd, G.D., Beale, R.: Human-Computer Interaction, 3rd edn. Prentice Hall, Harlow (2003)

9. Garrett, J.J.: The Elements of User Experience: User-Centered Design for the Web and Beyond, 2nd edn. New Riders Press, Berkeley (2010)

10. Google/OTX: 2010 Cruisers' Road to Decision, The role of Digital on Cruise Travelers (2010) 
11. Inversini, A., Cantoni, L.: Cultural Destination Usability: The Case of Visit Bath. In: Höpken, W., Gretzel, U., Law, R. (eds.) Information and Communication Technologies in Tourism 2009, pp. 319-331. Springer, Vienna (2009)

12. Inversini, A., Cantoni, L., Bolchini, D.: Presenting UsERA: User Experience Risk Assessment Model. In: Gretzel, U., Law, R., Fuchs, M. (eds.) Information and Communication Technologies in Tourism 2010, pp. 99-110. Springer, Vienna (2010)

13. Kuniavsky, M.: Observing the User Experience: A Practitioner's Guide to User Research, 1st edn. Morgan Kaufmann, San Francisco (2003)

14. Kuusela, H., Paul, P.: A Comparison of Concurrent and Retrospective Verbal Protocol Analysis. The American Journal of Psychology 113(3), 387-404 (2000)

15. Morgan, P.: FAT cruise tourism: The shifting tide of experience. In: Papathanassis, A. (ed.) Cruise Sector Growth: Managing Emerging Markets, Human Resources, Processes and Systems, pp. 43-57. Gabler, Wiesbaden (2009)

16. Nielsen, J.: Designing Web Usability, 1st edn. Peachpit Press (1999)

17. Nielsen, J.: Severity Ratings for Usability Problems (2006), http://www.useit. com/papers/heuristic/severityrating.html (April 2012)

18. Nielsen, J., Mack, R.L.: Usability Inspection Methods, 1st edn. Wiley, New York (1994)

19. O'Connor, P., Frew, A.J.: An evaluation methodology for hotel electronic channels of distribution. International Journal of Hospitality Management 23(2), 179-199 (2004)

20. Papathanassis, A., Breitner, M.: Online booking in the cruise sector: Determinants of online trust \& implications. In: Papathanassis, A. (ed.) Cruise Sector Growth: Managing Emerging Markets, Human Resources, Processes and Systems, pp. 133-154. Gabler, Wiesbaden (2009)

21. Poon, A.: Tourism, Technology and Competitive Strategies. CABI, Wallingford (1993)

22. Qi, S., Law, R., Buhalis, D.: A Study of Chinese and International Online User Perceptions of Hotel Websites Usefulness. In: Höpken, W., Gretzel, U., Law, R. (eds.) Information and Communication Technologies in Tourism 2009, pp. 285-296. Springer, Vienna (2009)

23. Reuters: Online travel sees offline touch for cruise (2008), http: / /www.reuters.com/article/2008/02/13/us-travel-leisuresummit-cruise-idUSN1338644520080213 (retrieved)

24. Triacca, L., Inversini, A., Bolchini, D.: Evaluating Web Usability with MiLE+. In: Proc. Seventh International Symposium on Web Site Evolution (WSE 2005), Budapest, pp. 22-29 (2005)

25. Werthner, H., Klein, S.: Information Technology and Tourism - A Challenging Relationship, 1st edn. Springer, Vienna (1999)

26. Yeung, T.A., Law, R.: Evaluation of Usability: A Study of Hotel Web Sites in Hong Kong. Journal of Hospitality \& Tourism Research 30(4), 452-473 (2006) 